\title{
Long-term outcome of flexible onabotulinum toxin A treatment in facial dystonia
}

\author{
John C Bladen ${ }^{1} \cdot$ Ilan Feldman ${ }^{1} \cdot$ Maribel Favor $^{1} \cdot$ Marizol Dizon $^{1} \cdot$ Andre Litwin $^{1} \cdot$ Raman Malhotra $^{1}$
}

Received: 18 October 2017 / Revised: 24 June 2018 / Accepted: 6 August 2018 / Published online: 10 September 2018

(c) The Royal College of Ophthalmologists 2018

\begin{abstract}
Purpose The purpose of this study was to assess the long-term outcome of onabotulinum used to treat facial dystonia and compare a flexible and fixed treatment regimen.

Methods This was a retrospective comparative study looking at benign essential blepharospasm (BEB), hemifacial spasm (HFS) and aberrant facial nerve regeneration synkinesis (AFR) treatment with onabotulinum toxin A (Botox $\left.{ }^{\circledR}\right)$ over a minimum of 10 years. Fifty-one patients were recruited into the study, with each dystonia subgroup having 17 patients. Blepharospasm disability score (BDS), subjective improvement score (SIS), duration of maximal effect (DME) and complications were recorded at each visit.

Results The mean age was 63 years and gender predominately female. Thirty-seven patients underwent flexible treatment intervals compared to 14 fixed treatment intervals, averaging 3.4 and 4 per annum, respectively. Mean BDS significantly improved from 6 to 3 at last review across all 3 groups, with the highest effect on BEB. BDS improvement was greater in flexible intervals. SIS remained similar for all three conditions during follow-up, but in those undergoing flexible intervals, SIS increased by a small margin compared to fixed interval. Mean DME was 10.5 weeks across all dystonias, but increased progressively only in the flexible interval group. Complications included ptosis (30\%), dry eye (14\%) and lagophthalmos $(8 \%)$.

Conclusion Flexible onabotulinum provided better long-term relief on BDS for facial dystonia than a fixed regimen. Flexible interval treatment may also provide better patient satisfaction and longer DME compared to fixed treatment. Both have similar complication rates. With flexible treatment however, fewer injections were required over 10 years, leading to cost saving.
\end{abstract}

\section{Introduction}

Benign essential blepharospasm (BEB) is a focal dystonia effecting the orbicularis muscle. Hemifacial spasm (HFS) is technically not a dystonia, but does cause twitching of the orbicularis oculi muscle that can spread to other facial muscles on the same side. HFS may be caused by irritation of the facial nerve such as an ectatic vessel crossing its root.

Electronic supplementary material The online version of this article (https://doi.org/10.1038/s41433-018-0203-3) contains supplementary material, which is available to authorized users.

John C Bladen

john.bladen1@nhs.net

1 Corneo Plastic Unit, Queen Victoria Hospital NHS Trust, East Grinstead, UK
Aberrant facial nerve regeneration (AFR) is a synkinesis whereby involuntary contractions of the facial muscles occurs when voluntary contraction of a different facial muscle group is instigated. Botulinum toxin was first approved for medical use on extraocular muscles to treat non-accommodative strabismus and, subsequently, efficacy was demonstrated in BEB, HFS and AFR [1-3]. At present, the accepted standard among physicians regarding interinjection interval for facial dystonia is generally 12 weeks or more. Nevertheless, the evidence for this regimen is weak [4]. A strict 3-monthly injection interval regimen may mean that patients experience a re-emergence of symptoms before a new injection is administered, thus resulting in dissatisfaction in the treatment cycle among patients. Moreover, Sethi et al. [5] reported that many patients who receive botulinum toxin type A for the treatment of cervical dystonia would prefer more frequent injections than the currently recommended 12-week inter-dose interval permits. 

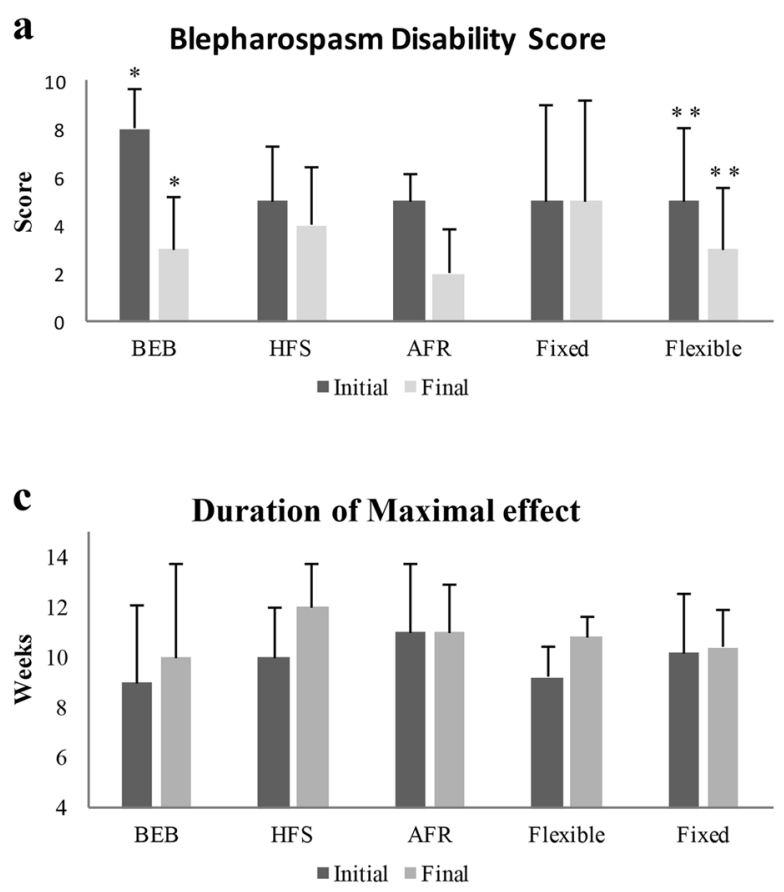

Fig. 1 Subjective and objective assessment of onabotulinum toxin A in blepharospasm (BEB), hemifacial spasm (HFS) and aberrance facial regeneration (AFR) during the first year of treatment (initial) compared to the last year of treatment (final). a Blepharospasm disability score. b Subjective improvement. c Duration of maximal effect.

Conversely, some patients have a sustained response beyond 3 months and, in strabismus cases, 6-monthly intervals can be effective [6-8].

In this study we report our 10-year experience of treating facial dystonia using onabotulinum toxin A and compare those who underwent a fixed versus a flexible treatment interval using both subjective and objective methods.

\section{Methods}

A retrospective chart review identified 17 patients for each facial dystonia group (51 patients) who had a minimum treatment period of 10 years (range 10-14) at a single centre (Queen Victoria Hospital, UK). Institutional review board approval was given and informed consent was collected from every patient. The criteria for inclusion were BEB, HFS or AFR treated with onabotulinum toxin A $\left(\right.$ Botox $\left.^{\circledR}\right)$ only. Allocation into the two groups was performed consecutively. Patients could choose whether to have fixed interval injections of 12 weeks or flexible intervals and were categorised accordingly (5, 12 BEB; 5, 12 HFS; and 4, 13 AFR respectively). Patients who remained in the same treatment regimen over a minimum of 10 years were included in the study. Flexible intervals involved a nursedriven clinic to be available every 2 weeks with a minimum of 2 slots reserved for patients to book in directly, up to a
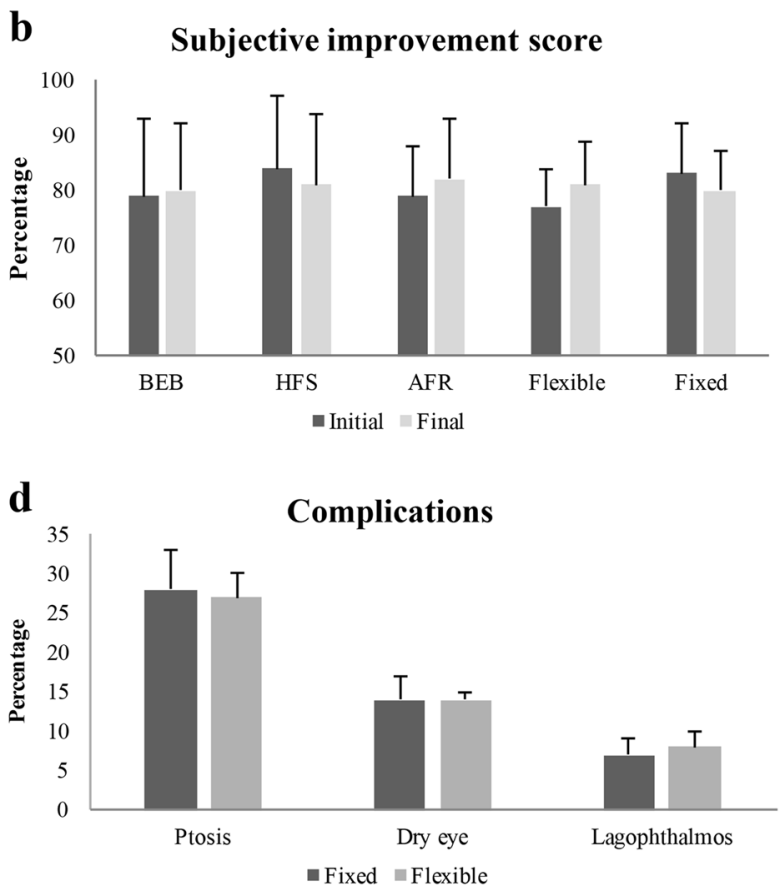

d Complication rate over the course of 10 years. Dystonias were grouped as follows: $n=17 \mathrm{BES}, n=17 \mathrm{HFS}$ and $n=17$ AFR Flexible group $n=37$ and fixed group $n=14$. Graphs represent mean values with error bars the standard deviation of the mean. $* P<0.01$, $* * P<0.05$

week prior. The system requires reserve, but all slots tend to be filled. Objective assessment included the blepharospasm disability score (BDS; supplementary Table S1) [9]. Subjective evaluation comprised the degree of improvement, treatment satisfaction (using a percentage rating scale of $0-100$ with $100 \%$ as very satisfied), duration of maximum effect (DME) in weeks and complications. All assessments were completed contemporaneously at each visit. Outcomes were summarised during the first year of treatment and at the final year of assessment to compare the initial and final response to toxin respectively. Progressive response between interval was also noted along with the number of injections required per year. Scrutiny for the presence of ptosis, strabismus, lagophthalmos and dry eye was performed at each visit; self-reporting by patients was also encouraged. $P$ values calculated using an unpaired $t$-test.

\section{Results}

The mean age of patients was 63 years (BEB 60, HFS 65 and AFR 64; range 31-88 years). In all, 14 males and 37 females were included. The interval of injections was flexible in 37 patients (72\%) and remained fixed in 14 (28\%). The number of mean annual injections for the flexible group was 3.4 and was 4 (12 weekly) for the fixed group over 10 years. Mean BDS improved significantly 
$(P=0.008)$ in the BEB group with a trend to improvement in HFS and AFR (Fig. 1a). BDS improvement mainly occurred in the first year (mean 3.4) with smaller fluctuations in subsequent years. In terms of treatment regimens, BDS improvement was greater in patients with flexible injection intervals (mean $2, P=0.045$ ) than those with fixed intervals which remained similar to initial visits. AFR $(P=0.067)$ demonstrated a trend to a significant improvement in BDS over the course of 10 years (Fig. 1a).

Subjective improvement remained similar for all three conditions during follow-up; however, mean subjective improvement in patients with flexible intervals increased by a small margin compared to those in fixed regimen (Fig. 1b).

DME increased progressively only in the flexible interval group by over 1 week, but remained static in the fixed cohort (Fig. 1c). The mean duration across all time points in all three was 10.5 weeks. An increase of 1 week was seen in the BEB, 2 weeks for HFS and no change for AFR (Fig. 1c) across the duration of the study.

The cumulative complication rate was the same in the flexible and fixed interval groups (Fig. 1d). Overall, up to $30 \%$ of patients experience ptosis as a side effect of their treatment at some point, as well as a significant risk of dry eye $(14 \%)$ or incomplete eye closure $(7-8 \%)$.

\section{Discussion}

This is the first study to demonstrate the long-term effectiveness of a flexible dosing regimen of onabotulinum toxin $A$ in the treatment of BEB, HFS and AFR. We found approximately an $80 \%$ subjective improvement in patient symptoms for all three facial dystonias. Moreover, BDS improvement mainly occurred in BEB and AFR over the course of treatment, and had a sustained response that was maintained for all three for a minimum of 10 years. Fewer injections were administered over the course of treatment in the flexible regimen compared to the fixed regimen, which confers a cost saving without compromising on satisfaction or efficacy. On the contrary, patients were more satisfied with a flexible regimen than those on a fixed schedule despite the total number of injections administered being less in the flexible group. Returning for a fixed appointment while the toxin is still therapeutic reduces the impact of the repeat dose and, if a repeat dose is refused, is a waste of clinical resources.

The DME was 10.5 weeks for all three facial dystonias, with the longest occurring in hemifacial spasm. Duration of maximal effect also increased in those patients undergoing a flexible dose regimen. Flexible treatment not only confers a cost advantage, but it also seems to be more efficacious than a fixed regimen, albeit marginally. The duration of effect depends on the condition being treated. Onabotulinum duration of effect for glabellar line treatment ranges between 10 and 24 weeks, although follow-up in these studies was short at 1 year or less [10-12]. The interval between injections increased with repeat doses of onabotulinum, with some waiting 7-10 months before a further dose, although retreatment interval may not be a good proxy for duration of effect [13]. A double-masked blepharospasm trial of Botox stated duration of effect as 8 weeks [14]. A study looking at blepharospasm, hemifacial spasm and cervical dystonia found the duration of effect to be $8.9,9.3$ and 9.2 weeks respectively [15]. A long-term study found a higher duration of action for blepharospasm and hemifacial spasm averaging 14.6 weeks, but mean duration of effect may not be synonymous with DME [16]. AFR treated with onabotulinum has been shown to last for 17.1 weeks but the maximal effect time was not stated [17]. Moreover, having a patient-led clinic will in itself improve the satisfaction of treatment as it ensures that not only are the users involved in shared decision making, but rather driving their own treatment.

Complication rates were higher than other reported studies, with ptosis affecting a third of patients. However, this is based on patients self-reporting ptosis from mild, visually insignificant to severe ptosis returning to clinic for a review. Ptosis is the most common side effect when treating BEB or HFS, with a long-term safety study stating a similar high risk of $40.6 \%$ [16]. Another long-term study using onabotulinum toxin A in blepharospasm reported a risk of ptosis as only $2.3 \%$ along with dry eye and diplopia as 0.5 and $0.2 \%$ respectively [18]. However, our adverse event outcome represents a cumulative risk of 10 years or more with patients encouraged to report any changes. It therefore gives a better overview of long-term treatment risk rather than some shorter studies that do not represent real-world outcomes [14, 18].

The weaknesses of this study include its retrospective nature. All questionnaires were however completed at the time of injection and patients were asked about DME and subjective improvement on each occasion, and so there is no recall bias. Case numbers were not large for each group and more studies looking at the long-term outcomes with larger numbers would be welcome. Moreover, teasing out the advantages and disadvantages of a fixed versus flexible treatment regimen would be beneficial for healthcare planning and costing.

In conclusion, flexible patient-led onabotulinum toxin A is effective for the long-term treatment of BEB, HFS and AFR with a maintained duration of effect. A flexible treatment regimen is better than fixed treatment regimen in terms of BDS and may be more beneficial subjectively, plus requires fewer injections over the course of 10 years, providing a cost saving. 


\section{Summary}

\section{What was known before}

- Onabotulinum toxin A has been shown to be beneficial in the treatment of facial dystonia using a fixed treatment regimen.

\section{What this study adds}

- Flexible onabotulinum toxin $\mathrm{A}$ is as efficacious as a fixed regimen in the treatment of three facial dystonias: blepharospasm, hemifacial spasm and aberrant facial regeneration synkinesis.

- Efficacy of each treatment remains similar even after 10 years.

- Flexible intervals may be more cost effective.

- This study is the first to report blepharospasm disability score outcomes for aberrant facial nerve regeneration synkinesis.

- Patient-led, nurse-driven botulinum clinics provide good patient experience which in turn is a measure of quality.

\section{Compliance with ethical standards}

Conflict of interest The authors declare that they have no conflict of interest.

\section{References}

1. Jankovic J, Orman J. Botulinum A toxin for cranial-cervical dystonia: a double-blind, placebo-controlled study. Neurology. 1987;37:616-23.

2. Clark RP, Berris CE. Botulinum toxin: a treatment for facial asymmetry caused by facial nerve paralysis. Plast Reconstr Surg. 1989;84:353-5.

3. Van den Bergh P, Francart J, Mourin S, Kollmann P, Laterre EC. Five-year experience in the treatment of focal movement disorders with low-dose Dysport botulinum toxin. Muscle Nerve. 1995;18:720-9.

4. Greene P, Fahn S, Diamond B. Development of resistance to botulinum toxin type A in patients with torticollis. Mov Disord. 1994;9:213-7.
5. Sethi KD, Rodriguez R, Olayinka B. Satisfaction with botulinum toxin treatment: a cross-sectional survey of patients with cervical dystonia. J Med Econ. 2012;15:419-23.

6. Hawamdeh ZM, Ibrahim AI, Al-Qudah AA. Long-term effect of botulinum toxin (A) in the management of calf spasticity in children with diplegic cerebral palsy. Eura Med. 2007;43:311-8.

7. Kanovsky P, Bares M, Severa S, Richardson A. Long-term efficacy and tolerability of 4-monthly versus yearly botulinum toxin type A treatment for lower-limb spasticity in children with cerebral palsy. Dev Med Child Neurol. 2009;51:436-45.

8. Cronemberger MF, Mendonca TS, Bicas HE. [Botulinum toxin treatment for horizontal strabismus in children with cerebral palsy]. Arq Bras Oftalmol. 2006;69:523-9.

9. Roggenkamper P, Jost WH, Bihari K, Comes G, Grafe S. Efficacy and safety of a new Botulinum Toxin Type A free of complexing proteins in the treatment of blepharospasm. J Neural Transm (Vienna). 2006;113:303-12.

10. Hankins CL, Strimling R, Rogers GS. Botulinum A toxin for glabellar wrinkles. Dose and response. Dermatol Surg. 1998;24:1181-3.

11. Carruthers J, Rivkin A, Donofrio L, Bertucci V, Somogyi C, Lei $\mathrm{X}$, et al. A multicenter, randomized, double-blind, placebocontrolled study to evaluate the efficacy and safety of repeated onabotulinumtoxinA treatments in subjects with Crow's feet lines and glabellar lines. Dermatol Surg. 2015;41:702-11.

12. Stotland MA, Kowalski JW, Ray BB. Patient-reported benefit and satisfaction with botulinum toxin type A treatment of moderate to severe glabellar rhytides: results from a prospective open-label study. Plast Reconstr Surg. 2007;120:1386-93. discussion 1394

13. Carruthers JD, Carruthers JA. Treatment of glabellar frown lines with C. botulinum-A exotoxin. J Dermatol Surg Oncol. 1992;18:17-21.

14. Nussgens Z, Roggenkamper P. Comparison of two botulinumtoxin preparations in the treatment of essential blepharospasm. Graefes Arch Clin Exp Ophthalmol. 1997;235:197-9.

15. Bihari K. Safety, effectiveness, and duration of effect of BOTOX after switching from Dysport for blepharospasm, cervical dystonia, and hemifacial spasm dystonia, and hemifacial spasm. Curr Med Res Opin. 2005;21:433-8.

16. Ababneh OH, Cetinkaya A, Kulwin DR. Long-term efficacy and safety of botulinum toxin A injections to treat blepharospasm and hemifacial spasm. Clin Exp Ophthalmol. 2014;42:254-61.

17. Toffola ED, Furini F, Redaelli C, Prestifilippo E, Bejor M. Evaluation and treatment of synkinesis with botulinum toxin following facial nerve palsy. Disabil Rehabil. 2010;32:1414-8.

18. Kollewe K, Mohammadi B, Kohler S, Pickenbrock H, Dengler R, Dressler D. Blepharospasm: long-term treatment with either Botox $(\mathrm{R})$, Xeomin(R) or Dysport(R). J Neural Transm (Vienna). 2015;122:427-31 